\title{
Temperature-stable operation of a quantum dot semiconductor disk laser
}

Cite as: Appl. Phys. Lett. 93, 051104 (2008); https://doi.org/10.1063/1.2968137

Submitted: 13 June 2008 • Accepted: 17 July 2008 • Published Online: 05 August 2008

T. D. Germann, A. Strittmatter, J. Pohl, et al.

\section{ARTICLES YOU MAY BE INTERESTED IN}

High-power semiconductor disk laser based on InAs / GaAs submonolayer quantum dots Applied Physics Letters 92, 101123 (2008); https://doi.org/10.1063/1.2898165

InAs/InP quantum dot VECSEL emitting at $1.5 \mu \mathrm{m}$

Applied Physics Letters 115, 171105 (2019); https://doi.org/10.1063/1.5125632

High-power InP quantum dot based semiconductor disk laser exceeding 1.3 W

Applied Physics Letters 102, 092101 (2013); https://doi.org/10.1063/1.4793299

四 QBLOX

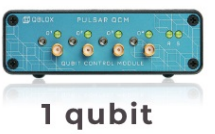

Shorten Setup Time Auto-Calibration More Qubits

Fully-integrated Quantum Control Stacks Ultrastable DC to $18.5 \mathrm{GHz}$ Synchronized $<<1 \mathrm{~ns}$ ultralow noise

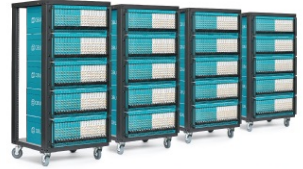

100s qubits

visit our website > 


\title{
Temperature-stable operation of a quantum dot semiconductor disk laser
}

\author{
T. D. Germann, ${ }^{1, a)}$ A. Strittmatter,${ }^{1}$ J. Pohl, ${ }^{1}$ U. W. Pohl, ${ }^{1}$ D. Bimberg, ${ }^{1}$ J. Rautiainen, ${ }^{2}$ \\ M. Guina, ${ }^{2}$ and O. G. Okhotnikov ${ }^{2}$ \\ ${ }^{1}$ Institut für Festkörperphysik, Sekr. EW 5-2, Technische Universität Berlin, Hardenbergstr. 36, 10623 \\ Berlin, Germany \\ ${ }^{2}$ Optoelectronics Research Centre, Tampere University of Technology, Korkeakoulunkatu 3, 33720 Tampere, \\ Finland
}

(Received 13 June 2008; accepted 17 July 2008; published online 5 August 2008)

\begin{abstract}
We demonstrate temperature-independent output characteristics of an optically pumped semiconductor disk laser (SDL) based on quantum dots (QDs) grown in the Stranski-Krastanow regime. The gain structure consists of a stack of $7 \times 3$ QD layers, each threefold group being located at an optical antinode position. The SDL emits at $1210 \mathrm{~nm}$ independent of the pump power density. Threshold and differential efficiency do not dependent on heat sink temperature. Continuous-wave operation close to $300 \mathrm{~mW}$ output power is achieved using the ground-state transition of the InGaAs QDs. (C) 2008 American Institute of Physics. [DOI: 10.1063/1.2968137]
\end{abstract}

Optically pumped semiconductor disk lasers, also referred to as vertical-external-cavity surface-emitting lasers (VECSELs), are very promising as high-power continuouswave $(\mathrm{cw})$ and short pulse light sources. ${ }^{1-5}$ Excellent, almost diffraction-limited beam quality with good power scalability ${ }^{1,6}$ was demonstrated for a wide spectral range accessible by efficient intra-cavity frequency upconversion. ${ }^{2,7,8}$ Future applications such as low-cost laser-based full-color video projection thus become feasible using well-established GaAs-based technology. Red color rendering for such systems requires infrared emission beyond $1200 \mathrm{~nm}$. In this wavelength range InGaAs quantum well (QW) structures face difficulties due to the large built-in strain. VECSELs for this wavelength range based on GaAsSb or GaInNAs QWs have been reported. ${ }^{1-3,6-10}$ However, strong temperature dependence of the emission wavelength is inherent to all QWbased VECSELs due to the spectrally comparatively small and temperature-dependent gain spectrum of QWs. Careful spectral detuning of the QW emission wavelength with respect to the cavity resonance at room temperature is required to obtain maximum output power at device operation temperatures ranging from 60 to $120^{\circ} \mathrm{C}$. Since the device temperature is influenced both by the incident pump power density as well as by heat dissipation via the heat sink and the heat spreader, device characteristics such as emission wavelength, threshold, and differential efficiency of QW-based VECSELs generally change depending on operation conditions. ${ }^{10}$ In contrast to QWs, quantum dots (QDs) prove a much broader gain spectrum as well as high thermal stability. ${ }^{11}$ QD-based lasers have demonstrated excellent performance in the spectral range from 980 to $1500 \mathrm{~nm} .^{12,13} \mathrm{Re}-$ cently, first QD-based VECSELs have been presented with $\mathrm{cw}$ operation at $1040 \mathrm{~nm}$ wavelength. ${ }^{5,14}$ The broad and flat gain spectrum of QDs offers the advantage of a temperatureindependent matching of the gain maximum with a given cavity resonance. Such characteristics are expected to result in temperature-independent device operation. In this letter, we present a VECSEL using the ground-state transitions of InGaAs QDs grown in the Stranski-Krastanow growth

${ }^{a)}$ Electronic mail: tgermann@ sol.physik.tu-berlin.de. mode. Indeed, output characteristics being independent of temperature in terms of threshold, differential efficiency, and the emission spectrum is demonstrated. First devices achieved $300 \mathrm{~mW} \mathrm{cw}$ output power at $1210 \mathrm{~nm}$ with a low threshold.

The active mirror of the VECSEL is composed of a semiconductor distributed Bragg reflector (DBR), the gain region consists of the QD layers, and a confinement window which prevents diffusion of the photoexcited charge carriers toward the surface. The structure was designed using the transfer-matrix method with material parameters taken from Ref. 15. The DBR consists of 37.5 pairs of $\mathrm{Al}_{0.98} \mathrm{Ga}_{0.02} \mathrm{As} / \mathrm{Al}_{0.2} \mathrm{Ga}_{0.8} \mathrm{As} \lambda / 4$ layers resulting in a spectral stop-band width of $80 \mathrm{~nm}$ and a nominal reflectivity of 99.96\%. Pump-light absorption by the gain-medium section takes place in the GaAs layers which enclose 21 QD layers. The thickness of the semiconductor gain medium is about $2.3 \mu \mathrm{m}$. From the characteristics of QD edge emitters based on similar dots a transparency current density of about $10 \mathrm{~A} / \mathrm{cm}^{2}$ per QD layer is calculated. ${ }^{16}$ Thus transparency of the whole stack is reached at incident power density levels well below $1 \mathrm{~kW} / \mathrm{cm}^{2}$. Defect-free QDs are essential since defect multiplication at successive QD layers may easily occur upon stacking with such large layer numbers, leading to rapidly degrading device performance. Furthermore, for QD layer spacings below $\sim 40 \mathrm{~nm}$ strain-induced structural coupling between individual QD layers leads to increased inhomogeneous broadening upon stacking due to increased QD sizes. ${ }^{17}$ Requirements for uniform defect-free dots are met for a minimum spacer thickness of $45 \mathrm{~nm}$ as proven by photoluminescence (PL) spectroscopy and inspection of the surface morphology. Such spacer thickness allows for three symmetrically positioned QD layers per antinode. Given a modal gain value of $\sim 5 \mathrm{~cm}^{-1}$ per QD layer for the groundstate transition ${ }^{18,19}$ for similar layers modeling shows that lasing can be achieved with 21 QD layers using a highly reflecting $(R>99.8 \%)$ output coupling mirror. The topmost carrier-diffusion barrier is realized by a lattice-matched $65 \mathrm{~nm}$ thick $\mathrm{In}_{0.48} \mathrm{Ga}_{0.53} \mathrm{P}$ layer which is transparent to the pump light and does not require an absorbing GaAs oxidation-protection layer. 


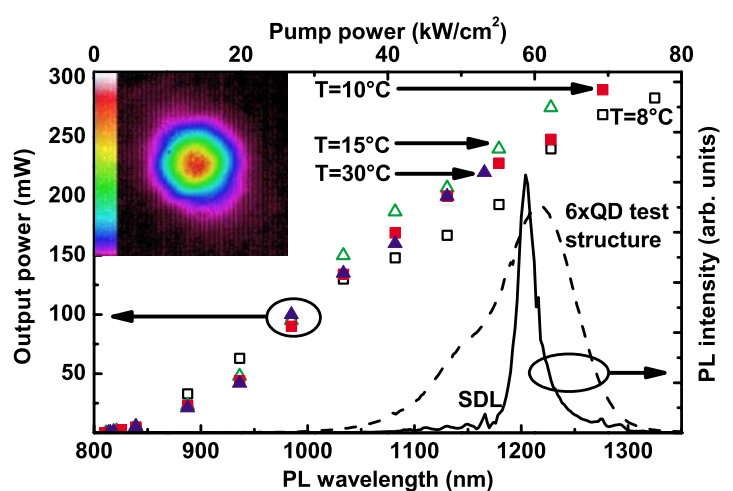

FIG. 1. (Color online) Left and top axes: light output characteristics with heat sink set to different temperatures. Right and bottom axes: PL of sixfold QD test structure and surface PL of QD VECSEL excited at $532 \mathrm{~nm}$ with excitation densities of 300 and $30 \mathrm{~W} / \mathrm{cm}^{2}$, respectively. Inset: spatial intensity profile of the output beam recorded at $170 \mathrm{~mW}$ output power.

The devices are grown on Si-doped GaAs 2 in. substrates using metal-organic vapor phase epitaxy. After deoxidizing the wafers at $715{ }^{\circ} \mathrm{C}$ under arsine, a GaAs buffer layer, the DBR structure, and $130 \mathrm{~nm}$ GaAs of the subcavity are grown at $680^{\circ} \mathrm{C}$. Subsequently, the temperature is cycled between $500{ }^{\circ} \mathrm{C}$ for QD growth with tertiarybutylarsine and $595{ }^{\circ} \mathrm{C}$ for $\mathrm{GaAs}$ spacer growth with arsine. The final $\mathrm{In}_{0.48} \mathrm{Ga}_{0.52} \mathrm{P}$ window layer is grown using tertiarybutylphosphine at $595{ }^{\circ} \mathrm{C}$. In order to tune the QD emission wavelength to $1210 \mathrm{~nm}$, the $\mathrm{In}_{0.68} \mathrm{Ga}_{0.32} \mathrm{As}$ QDs are overgrown by a $3.9 \mathrm{~nm}$ thick $\mathrm{In}_{0.12} \mathrm{Ga}_{0.88} \mathrm{As}$ layer. Details of QD growth are given in Ref. 20. Optical reflectance spectra recorded by an optical spectrum analyzer are used to adjust the DBR stop band and the cavity length. To characterize QD and VECSEL emission room temperature PL measurements are performed on PL test structures and on complete VECSEL gain chips with excitation by a frequency-doubled Nd:YAG (yttrium aluminum garnet) laser at $532 \mathrm{~nm}$. The PL test structures comprise two AlGaAs diffusion barriers enclosing the QD layers in the $300 \mathrm{~nm}$ GaAs matrix.

To investigate the stacking properties of the QDs, PL test structures with up to six QD layers are characterized (Fig. 1). Using recently developed, improved growth conditions for cap and spacer layers ${ }^{16}$ neither broadening nor shifting of the ground-state emission upon stacking is observed in the PL spectrum. This demonstrates the excellent overgrowth stability of the QD emission.

To assess VECSEL operation characteristics a $267 \mu \mathrm{m}$ thick natural diamond heat spreader is liquid capillary bonded onto the surface of a $2.5 \times 2.5 \mathrm{~mm}^{2}$ gain-mirror piece. The bonded wafer is mounted on a water-cooled copper heat sink kept at $15{ }^{\circ} \mathrm{C}$ unless otherwise mentioned. Optical pumping is provided by a fiber-coupled diode laser delivering up to $50 \mathrm{~W} \mathrm{cw}$ power at $790 \mathrm{~nm}$, incident at $35^{\circ}$ to the surface normal. Pump-spot size on the gain-chip surface and the size of the cavity mode are matched to a diameter of $180 \mu \mathrm{m}$. The external V-shaped cavity is realized by a focusing mirror with a radius of curvature of $200 \mathrm{~mm}$, a high reflectivity (99.8\%) output-coupler mirror, and the gain chip itself. A very low pump power threshold density of $2-3 \mathrm{~kW} / \mathrm{cm}^{2}$ is achieved for all temperatures which is twice lower than recently reported for InGaAsN-QW based VECSELs. ${ }^{9}$ Threshold pump power is $0.48 \mathrm{~W}$ at $15{ }^{\circ} \mathrm{C}$ (Fig. 2 ). The Gaussian intensity profile of the laser beam (inset of

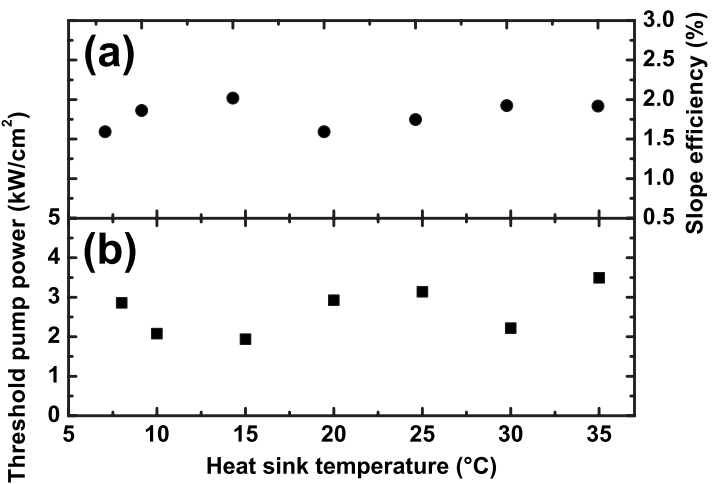

FIG. 2. (a) Slope efficiency of the VECSEL and (b) threshold pump power both measured with copper heat sink set to different temperatures.

Fig. 1) as recorded by a charge-coupled device camera at $170 \mathrm{~mW}$ output power indicates nearly diffraction-limited emission of the VECSEL. A maximum output power close to $300 \mathrm{~mW}$ is achieved for heat sink temperatures of up to $15{ }^{\circ} \mathrm{C}$, being limited by the thermal rollover. With higher heat sink temperatures leading to thermal rollover comes up at lower temperatures (Fig. 1) but no degradation was observed for hours of operation at high pump power level. A temperature-independent differential efficiency of $2 \%$ is found. The low value results from the limited modal gain of the structure. Modeling suggests that a lower output coupling mirror reflectivity of $98 \%$ is sufficient for 30 grouped QD layers in order to achieve lasing. Emission spectra recorded at different heat sink temperatures (for a medium pump power level) shown in Fig. 3 demonstrate an excellent center wavelength stability of $0.06 \mathrm{~nm} / \mathrm{K}$. The value corresponds to the shift of the cavity resonance. In comparison, typical values of GaInNAs-QW based VECSELs show a shift of $0.3 \mathrm{~nm} / \mathrm{K} .^{10}$ Emission-wavelength stability is proven by the almost constant center wavelengths in the spectra recorded at different output powers, cf. Figs. 4(a)-4(e). The dependence on pump power density is calculated to be $0.027 \mathrm{~nm} /(\mathrm{kW}$ $\times \mathrm{cm}^{-2}$ ) independent of the heat sink temperature. This is in agreement with an almost constant peak wavelength of the PL emission shown in Fig. 4(f). A shift of only $2 \mathrm{~nm}$ and a broadening of $1 \mathrm{~nm}$ of full width at half maximum are found

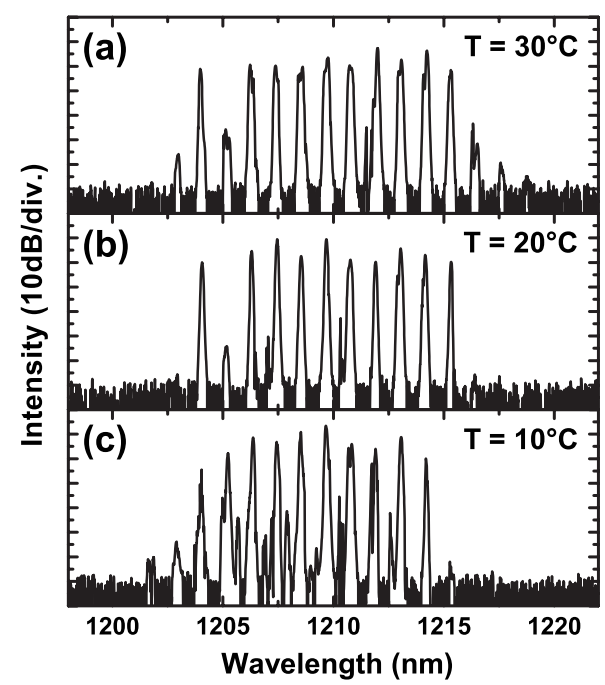

FIG. 3. [(a)-(c)] Laser spectra of the VECSEL recorded at a medium pump power level of $30 \mathrm{~kW} / \mathrm{cm}^{2}$ with heat sink set to different temperatures. 


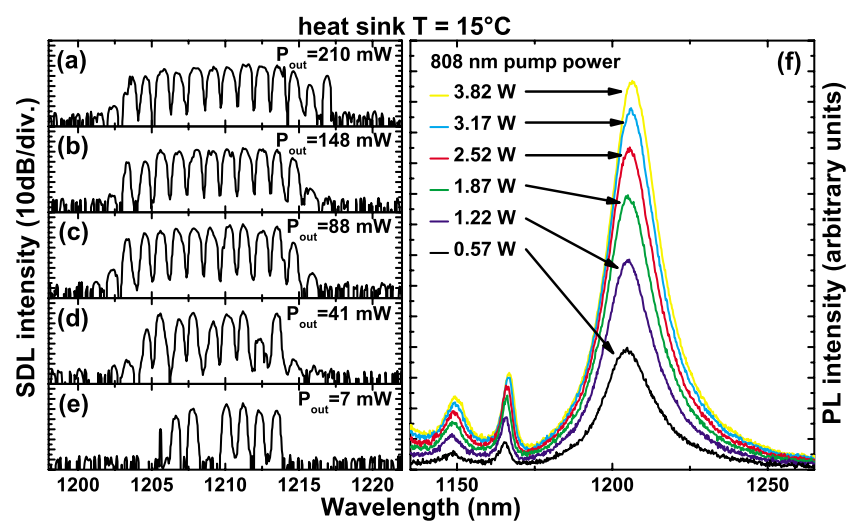

FIG. 4. (Color online) [(a)-(e)] Laser spectra recorded at different output power levels with heat sink temperature set to $15{ }^{\circ} \mathrm{C}$. (f) PL spectra recorded in a different VECSEL setup at different pump-power levels below lasing threshold.

as the pump power is increased from 30 to $190 \mathrm{~kW} / \mathrm{cm}^{2}$. The shift corresponds to the resonance shift of the VECSEL.

In conclusion, we demonstrate temperature-independent ground-state $\mathrm{cw}$ lasing of a QD VECSEL at $1210 \mathrm{~nm}$. Stable laser characteristics in terms of threshold, differential efficiency, and emission spectrum, being independent of the incident pump power and the heat sink temperature is achieved employing the broad and flat gain spectrum of a 21-fold InGaAs QD stack. The VECSEL shows stable operation without indications of degradation, a Gaussian beam profile, and close to $300 \mathrm{~mW}$ output power.

The authors acknowledge support from EU FP6 NATAL Contract No. 016769 and Sfb 787 funded by DFG.

${ }^{1}$ M. Kuznetsov, F. Hakimi, R. Sprague, and A. Mooradian, IEEE J. Sel.
Top. Quantum Electron. 5, 561 (1999).

${ }^{2}$ J. Lee, S. Lee, T. Kim, and Y. Park, Appl. Phys. Lett. 89, 241107 (2006).

${ }^{3}$ P. Klopp, F. Saas, M. Zorn, M. Weyers, and U. Griebner, Opt. Express 16 5770 (2008).

${ }^{4}$ D. Lorenser, D. J. H. C. Maas, H. J. Unold, A. R. Bellancourt, B. Rudin, E. Gini, D. Ebling, and U. Keller, IEEE J. Quantum Electron. 42, 838 (2006)

${ }^{5}$ T. D. Germann, A. Strittmatter, J. Pohl, U. W. Pohl, D. Bimberg, J. Rautiainen, M. Guina, and O. G. Okhotnikov, Appl. Phys. Lett. 92 101123 (2008).

${ }^{6}$ A. C. Tropper, H. D. Foreman, A. Garnache, K. G. Wilcox, and S. H. Hoogland, J. Phys. D 37, R75 (2004).

${ }^{7}$ E. Gerster, I. Ecker, S. Lorch, C. Hahn, S. Menzel, and P. Unger, J. Appl. Phys. 94, 7397 (2003)

${ }^{8}$ A. Harkonen, J. Rautiainen, M. Guina, J. Konttinen, P. Tuomisto, L. Orsila, M. Pessa, and O. G. Okhotnikov, Opt. Express 15, 3224 (2007).

${ }^{9}$ W. Diehl, P. Brick, B. Kunert, S. Reinhard, K. Volz, and W. Stolz, Appl. Phys. Lett. 91, 071103 (2007).

${ }^{10}$ J. Konttinen, A. Harkonen, P. Tuomisto, M. Guina, J. Rautiainen, M Pessa, and O. Okhotnikov, New J. Phys. 9, 140 (2007).

${ }^{11}$ M. Grundmann, O. Stier, S. Bognar, C. Ribbat, F. Heinrichsdorff, and D. Bimberg, Phys. Status Solidi A 178, 255 (2000).

${ }^{12}$ D. Bimberg and N. Ledentsov, J. Phys.: Condens. Matter 15, R1063 (2003).

${ }^{13}$ D. Bimberg, Electron. Lett. 44, 168 (2008).

${ }^{14}$ A. Strittmatter, T. D. Germann, J. Pohl, U. W. Pohl, D. Bimberg, J. Rautiainen, A. Guina, and O. G. Okhotnikov, Electron. Lett. 44, 290 (2008).

${ }^{15}$ S. Adachi, J. Appl. Phys. 58, R1 (1985).

${ }^{16}$ T. D. Germann, A. Strittmatter, T. Kettler, K. Posilovic, U. W. Pohl, and D. Bimberg, J. Cryst. Growth 298, 591 (2007).

${ }^{17}$ B. Lita, R. S. Goldman, J. D. Phillips, and P. K. Bhattacharya, Appl. Phys. Lett. 74, 2824 (1999).

${ }^{18}$ N. Kirstaedter, O. G. Schmidt, N. N. Ledentsov, D. Bimberg, V. M Ustinov, A. Y. Egorov, A. E. Zhukov, M. V. Maximov, P. S. Kopev, and Z I. Alferov, Appl. Phys. Lett. 69, 1226 (1996).

${ }^{19}$ L. F. Lester, A. Stintz, H. Li, T. C. Newell, E. A. Pease, B. A. Fuchs, and K. J. Malloy, IEEE Photonics Technol. Lett. 11, 931 (1999).

${ }^{20}$ R. L. Sellin, I. Kaiander, D. Ouyang, T. Kettler, U. W. Pohl, D. Bimberg, N. D. Zakharov, and P. Werner, Appl. Phys. Lett. 82, 841 (2003). 